$\xi=-1$

\title{
Facial thermography: a potential complimentary tool for evaluation of dental disorders
}

\author{
U. Snekhalatha ${ }^{1}{ }^{*}$, Nida Mir ${ }^{1}$, Mehvish Khan ${ }^{1}$, Parimal Raj ${ }^{1}$, Vimaladhithan ${ }^{1}$, Yeshi Choden ${ }^{1}$ \\ ${ }^{1}$ Department of Biomedical Engineering, SRM University, Kattankulathur, chennai-603203, Tamilnadu, India \\ *Corresponding author E-mail: sneha_samuma@yahoo.co.in
}

\begin{abstract}
The aim and objectives of the study was to analyze the skin surface temperature distribution in dental disorders using thermal imaging and to segment the region of interest using k-means algorithm and to perform statistical feature extraction for the total population studied. Thermal images were obtained for left profile, right profile, and front profile for the total population to be studied. The thermal image was segmented using k-means algorithm and the features were extracted from the segmented output image for both normal and dental disorder subjects. The results obtained from the study depicts that the mean temperature difference between the abnormal diseased subjects and the healthy controls in the front, left and right facial region was found to be $8.75 \%, 7.89 \%$ and $9.3 \%$ respectively. Among the regions examined in the facial thermogram, right facial region depicts the highest percentage difference of $9.3 \%$ found between the diseased subjects and normal group. From this data it is safe to infer that, presence of infections, significantly increases the temperature of the region. Hence thermography was used as a complimentary diagnostic tool in diagnosis of dental disorders.
\end{abstract}

Keywords: Thermography; K-Means Algorithm; Feature Extraction; Facial-Thermogram; Dental Diseases.

\section{Introduction}

Periodontal disease refers to the infections in and around the structures of the teeth which includes gums, cavities, ligament, alveolar bone and cementum that covers the root. Worldwide, around $11.2 \%$ people were affected by periodontitis [1]. The prevalence rate of dental disorder in India was $85 \%$ in their general populations [2]. According to World health organization report, it was estimated that around $60-90 \%$ of children and $100 \%$ adults suffering from dental cavities problem. Peridontal disease affects the 15$20 \%$ of adult people whose age varies between 35-44 years [3]. Along with these disorders, people suffer from asymptomatic diseases or Temporomandibular Joint (TMJ) disorders, which might go unnoticed or undiagnosed. Conventional diagnostic methods include clinical intra and extra-oral examination, radiographic method, occlusal examination and fiber-optic Transillumination, Electrical conductance, quantitative light induced fluoroscope [4], [5]. The non- invasive complimentary diagnostic method used for dental examination is thermal imaging method.

Our human body has a tendency to produce heat through the tissues by means of conduction, convection, and emits the body temperature through the skin. The heat transfer from skin to the air by means of infrared radiation, the radiant intensity will be received by the infrared cameras. Thus the infrared cameras produce the thermal imaging from the area of interest. Hence thermal imaging is used for studying the physiological characteristics related to the skin temperature [6] and serve as a non invasive imaging modality for diagnosing the dental disorders.

Gratt et al measured the facial skin temperature using static area telethermography for the diagnosis of chronic orofacial pain and TMJ disorders. They found the correlation between the orofacial pain and thermal abnormalities in the facial regions [7]. Gratt et al in their study conducted the clinical assessment of dental disorders for six years and performed thermal classification system to differentiate the peripheral nerve mediated pain, TMJ arthropathy, maxillary sinusitis and psychogenic facial pain using electronic facial thermography. They predicted that $92 \%$ accuracy attained for classification between the patients with orofacial pain and normal subjects [8]. Hayase et al conducted the clinical study to evaluate the malignant tumors in oral and maxillo-facial region using facial thermography. They measured the skin temperature difference between the affected region and opposite normal region and observed the higher temperature in malignant tumors regions [9]. Several researchers used facial thermography as a potential diagnostic tool for the evaluation of diseases in maxillofacial areas [10], [11]. Hence facial thermography could be used as complementary diagnostic tool for the evaluation of various dental disorders. Image segmentation techniques are very important in segregating the region of hot spot abnormal regions which depicts the dental disorders. The aim and objectives of the study was to analyze the skin surface temperature distribution in dental disorders using thermal imaging and to segment the region of interest using $\mathrm{k}$-means algorithm and to perform statistical feature extraction for the total population studied.

\section{Subjects and methods}

\subsection{Study design and population}

The institutional scientific committee of SRM Dental Hospital had approved the study, and the participants signed the informed consent form. A total number of 42 subjects, were considered, out of which 10 were normal, and rest 32 included patients with a history of Temporomandibular joint disorders (TMD), asymptomatic orodental infections and pain, which could be imaged using thermal camera were considered for the study. Subjects with beard, or 
wearing ornaments or jewelry near the region of interest that could not be removed were excluded.

\subsection{Thermal image analysis procedure}

The acquisition of thermal image was performed according to the guidelines recommended by the International Academy of Clinical Thermology. All the subjects were made to remove all the ornaments within the region of interest and were seated in a temperature-controlled room at $20{ }^{\circ} \mathrm{C}$ with humidity of $45 \%-50 \%$. The thermal camera was positioned at a distance of $1.0 \mathrm{~m}$ and a thermal image of left, right and front views of the face were taken. All the thermal images were acquired in the same room [12]. A hand-held thermal camera (FLIR, A300) was used to image the left, right and front views of the face region of both the subject groups (normal and diseased). The thermal camera FLIR A300 utilizes the $320 X 420$ thermal element focal plane array (FPA) uncooled microbolometer detector system with minimum focusable distance of $0.4 \mathrm{~m}$ to infinity. The camera could measure the temperature range of $220{ }^{\circ} \mathrm{C}$ to $1200{ }^{\circ} \mathrm{C}$ to an accuracy of $2 \%$ with thermal sensitivity of $0.05{ }^{\circ} \mathrm{C}$ [12]. The images were stored and then analyzed using the software FLIR TOOLS Software and further processed with MATLAB R2012a (MathWorks Inc., Natick, MA, USA). From the thermal images, the average skin temperature $\left({ }^{\circ} \mathrm{C}\right)$ was measured by positioning an available rectangle area tool of ROI on each respective region of interest.

\subsection{Thermal image segmentation algorithm}

A summary of the segmentation algorithm was given below:

a) The images of facial region showing the dental abnormalities were acquired and pre-processed.

b) The images was converted from RGB Colour Space to $\mathrm{L}^{*} \mathrm{a} \mathrm{b}^{*}$ Colour Space where $\mathrm{L}$ is lightness where its value varies from 0 to 100 , 'a' refers to green-red component and 'b' refers to blue-yellow component.

c) Three different clusters were created to segment the hot spot high temperature region in dental abnormalities d) The colours were Classified in ' $a * b *$ ' Space Using K-Means Clustering.

e) Each pixel in the Image was labelled using the results from K-Means algorithm.

\section{Results}

The mean temperature for dental abnormalities had elevated temperature compared to the normal subjects. The mean temperature for the normal group which was calculated was found out to be $\cong 31{ }^{\circ} \mathrm{C}$ and that of abnormal group was calculated to be $\cong 34{ }^{\circ} \mathrm{C}$ which $\cong 3{ }^{\circ} \mathrm{C}$ more than normal subjects. The sites of discomfort varied; in some cases, the symptoms were experienced over and around the masseter muscle and inflammation was reported in the affected region. Table 1 represents the skin temperature distribution in facial region for normal and dental disorder. The use of infrared thermography enabled assessment of the maximal temperature for each dental disorder. The features such as mean, standard deviation, variance, skewness, kurtosis and moment were extracted for normal and diseased subjects (table 2). It was found that these parameters for normal subjects were zero and for abnormal subjects the values were elevated.

Table 1 depicts the mean of maximum, minimum and average temperatures that were recorded from the front, left and right profiles of the normal and diseased subjects. The average temperature difference between the abnormal diseased subjects and the normal subjects in the front, left and right facial region was found to be $8.75 \%, 7.89 \%$ and $9.3 \%$ respectively. Among the regions examined in the facial thermogram, right facial region depicts the highest percentage difference of $9.3 \%$ found between the diseased subjects and normal group. From the table, it is easy to infer that the average temperature of front, left and right profiles, recorded at the region of interest, of the diseased patients is always higher in comparison to that of normal subjects. An approximate elevation of $3^{0} \mathrm{C}$ was observed between the normal and diseased subjects.

Table 1: Skin Temperature Distribution in Facial Region for Normal and Dental Disorder

\begin{tabular}{llllll}
\hline Facial Region & Temperature & Normal $(\mathrm{n}=10)$ Mean \pm std & Diseased $(\mathrm{n}=32)$ Mean \pm std & $\%$ difference & Statistical significance \\
\hline \multirow{3}{*}{ Front } & Max & $36.1 \pm 0.5$ & $36.53 \pm 0.3$ & 1.1 & 0.05 \\
& Min & $27.07 \pm 1.1$ & $31.67 \pm 0.2$ & 8.75 & 0.001 \\
& Mean & $31.585 \pm 0.7$ & $34.61 \pm 0.2$ & 3.4 & 0.003 \\
Left & Max & $35.22 \pm 0.7$ & $36.46 \pm 0.3$ & 11.06 & 0.001 \\
& Min & $27.98 \pm 1.04$ & $31.46 \pm 0.5$ & 7.89 & 0.004 \\
\multirow{4}{*}{ Right } & Mean & $31.6 \pm 0.67$ & $34.31 \pm 0.5$ & 3.2 & 0.02 \\
& Max & $35.31 \pm 0.62$ & $36.48 \pm 0.3$ & 14.33 & 0.001 \\
& Min & $27.07 \pm 1.5$ & $31.6 \pm 0.4$ & 9.3 & 0.002 \\
\hline
\end{tabular}

Table 2: Feature Extracted Parameters of Facial Regions for Normal and Diseased Subjects

\begin{tabular}{|c|c|c|c|c|c|c|}
\hline \multirow{2}{*}{ Feature extracted parameters } & \multicolumn{3}{|c|}{$\begin{array}{l}\text { Normal }(n=10) \text { Mean } \pm \text { Std } \\
\text { Facial Region }\end{array}$} & \multicolumn{3}{|c|}{$\begin{array}{l}\text { Diseased }(n=32) \text { Mean } \pm \text { Std } \\
\text { Facial Region }\end{array}$} \\
\hline & Front & Left & Right & Front & Left & Right \\
\hline Mean & 0 & 0 & 0 & $0.007528 \pm 0.003$ & $0.0079 \pm 0.003$ & $0.0095 \pm 0.007$ \\
\hline Std dev & 0 & 0 & 0 & $0.1116 \pm 0.2$ & $0.08627 \pm 0.02$ & $0.08919 \pm 0.02$ \\
\hline Variance & 0 & 0 & 0 & $0.0069 \pm 0.003$ & $0.0079 \pm 0.003$ & $0.0084 \pm 0.004$ \\
\hline Skewness & 0 & 0 & 0 & $17.9963 \pm 24.8$ & $11.6798 \pm 3.69$ & $12.4958 \pm 7.62$ \\
\hline Kurtosis & 0 & 0 & 0 & $920.374 \pm 3152.4$ & $150.2 \pm 133.8$ & $209.912 \pm 445$ \\
\hline Moment & 0 & 0 & 0 & $0.0069 \pm 0.003$ & $0.0079 \pm 0.003$ & $0.0084 \pm 0.004$ \\
\hline
\end{tabular}

Table 2 depicts the statistical calculations carried out on the collected data, which includes mean, standard deviation, skewness, kurtosis and moment of the temperature values collected from the front, left and right profiles of the normal and diseased subjects. These values are evidently zero incase of normal subjects, unlike those seen in diseased subjects.

Figure 1 shows the front profile of the normal subjects on which K-Means clustering is applied. Specifically Figure (1a) shows the Maximum and Minimum temperature zones which are located using FLIR Software, being the input image, along with the highest temperature, lowest temperature and the average temperature.
Figure (1b) is the image with its cluster index displayed as gray image. Figure (1c), displays the first cluster of three clusters separately, in which the image is partitioned, depicting the background. Figure (1d), displays the second cluster of three clusters separately, in which the image is partitioned, depicting the other regions. Figure (1e), displays the third cluster of three clusters separately, in which the image is partitioned, displaying the high temperature regions, depicting inflammation and presence of infection, (not seen in the thermogram of normal subject). Figure (1f) shows the segmentation of maximum temperature zone using k-means clustering and thresholding. Since there is no high tem- 
perature zone is present in the front profile image of a normal subject, high temperature zone is not segmented in this image. similarly figure (2a)-(2f), figure (3a)-(3f), figure (4a)-(4f), figure (5a)-(5f), figure (6a)-(6f) indicates the various stages of segmenta- tion process of normal left, normal right, diseased front, diseased left , diseased right profile respectively.

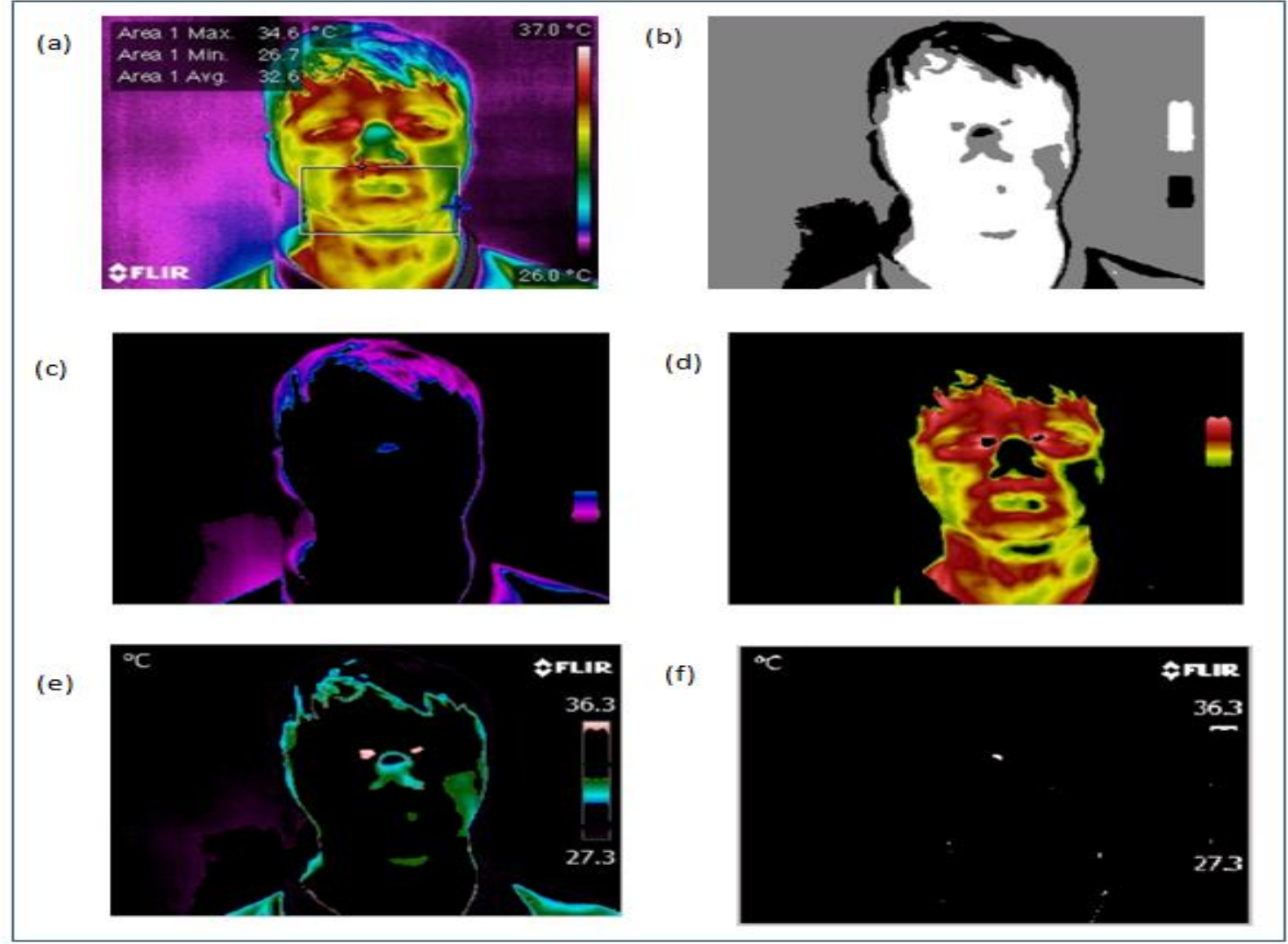

Fig. 1: Normal Subject Front Profile(A)Input Thermal Image,(B) Image with Its Cluster Index,(C) Cluster 1 Depicting the Background, (D) Cluster 2 Depicting other Regions, (E) Cluster 3 Depicting High Temperature Regions ,(F) Segmented Image (Absence of High Temperature).

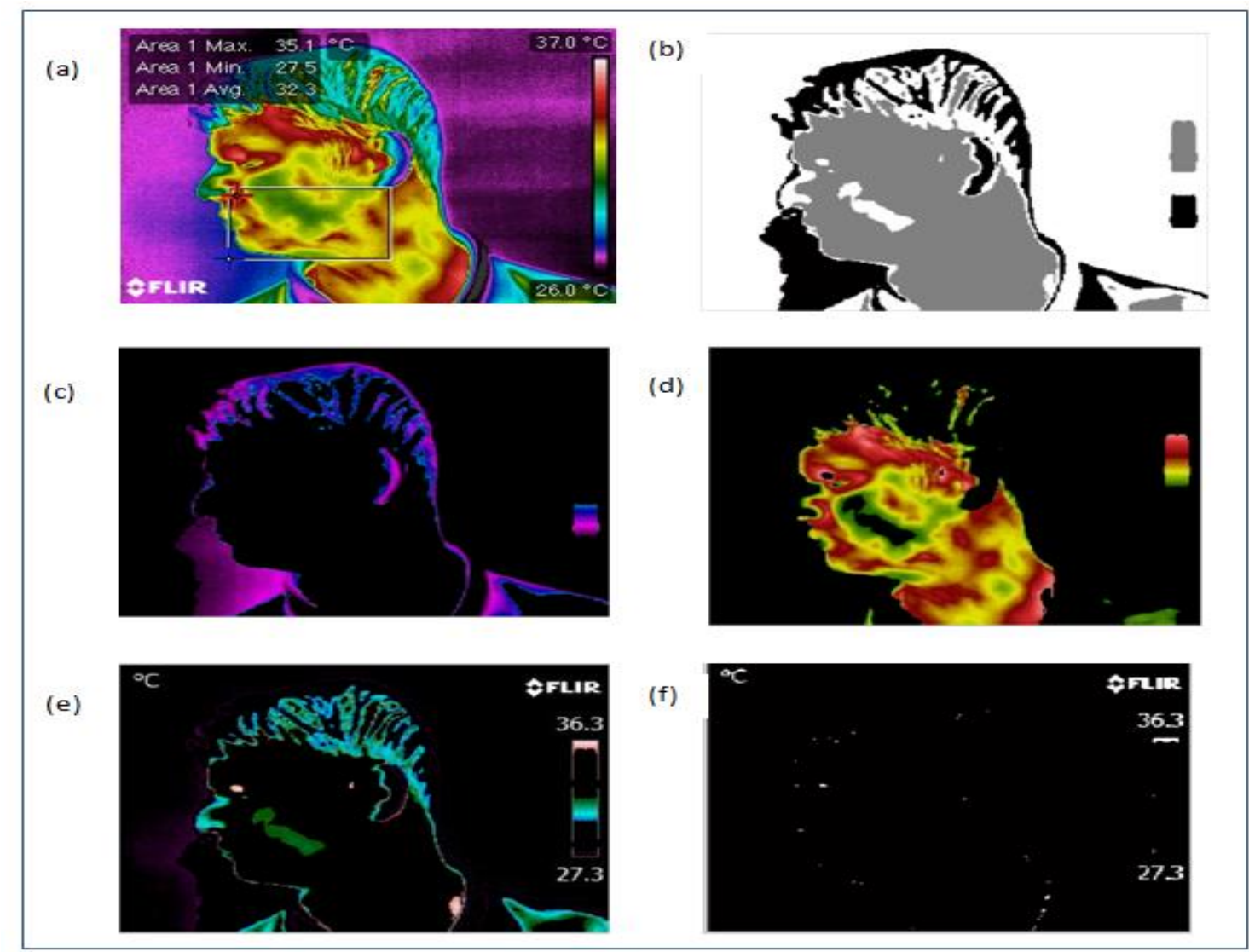

Fig. 2: Normal Subject Left Profile (A)Input Thermal Image, (B) Image with Its Cluster Index,(C) Cluster 1 Depicting the Background, (D) Cluster 2 Depicting other Regions, (E) Cluster 3 Depicting High Temperature Regions, (F) Segmented Image (Absence of High Temperature). 


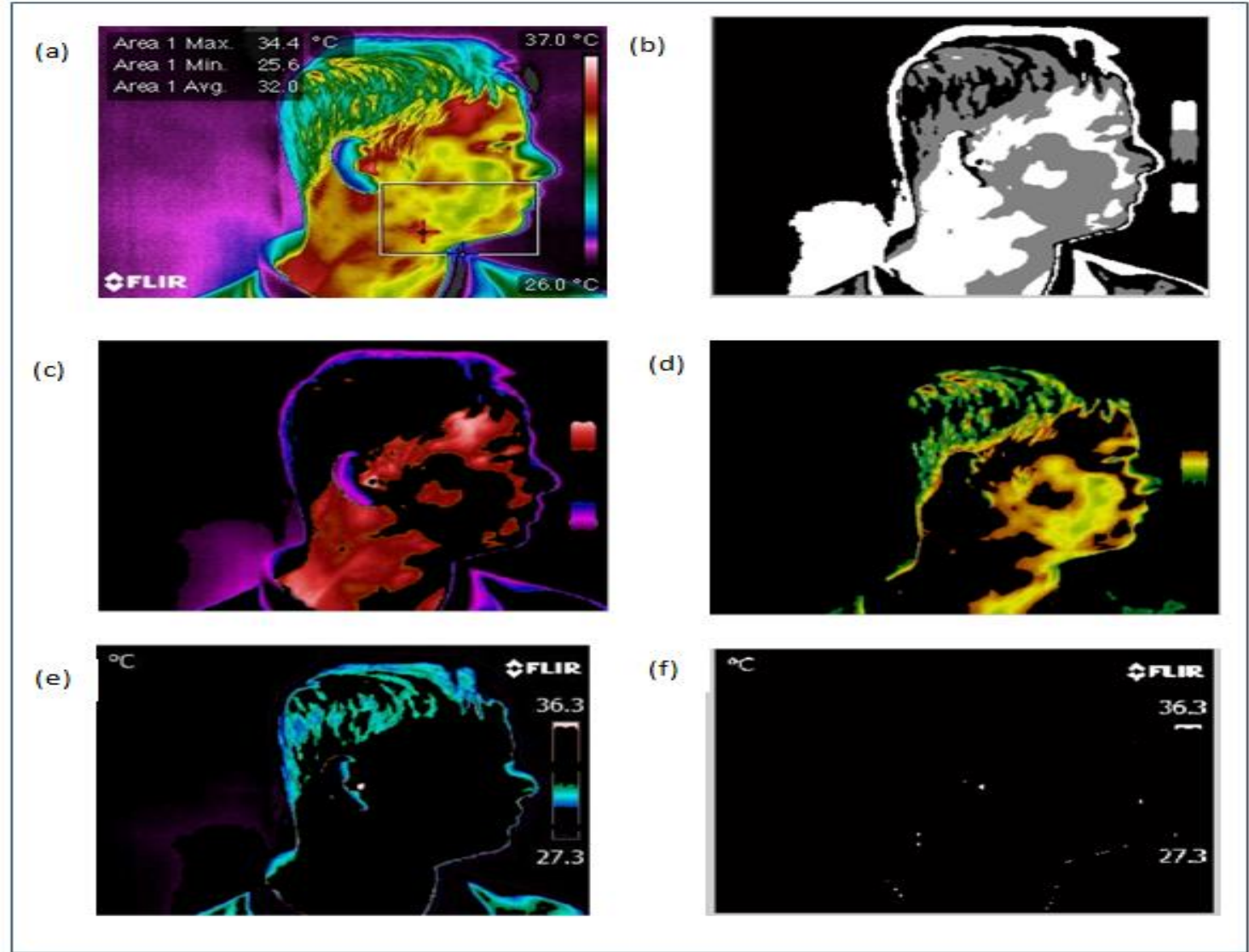

Fig. 3: Normal Subject Right Profile (A) Input Thermal Image, (B) Image with Its Cluster Index, (C) Cluster 1 Depicting the Background, (D) Cluster 2 Depicting other Regions, (E) Cluster 3 Depicting High Temperature Regions, (F) Segmented Image (Absence of High Temperature).

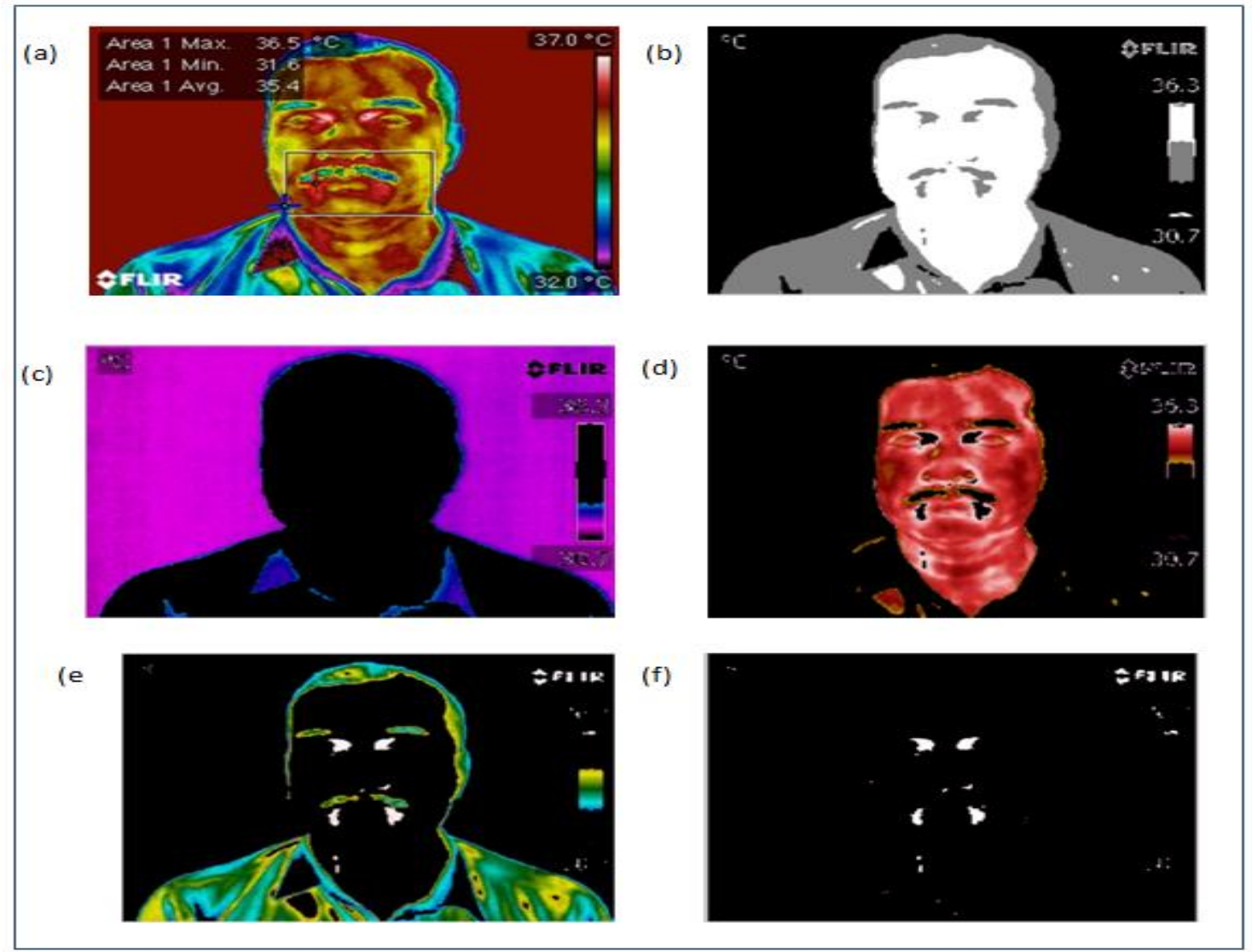

Fig. 4: Diseased Subject Front Profile (A) Input Thermal Image, (B) Image with Its Cluster Index, (C) Cluster 1 Depicting the Background, (D) Cluster 2 Depicting other Regions, (E) Cluster 3 Depicting High Temperature Regions, (F) Segmented Image Depicting Only High Temperature Regions. 


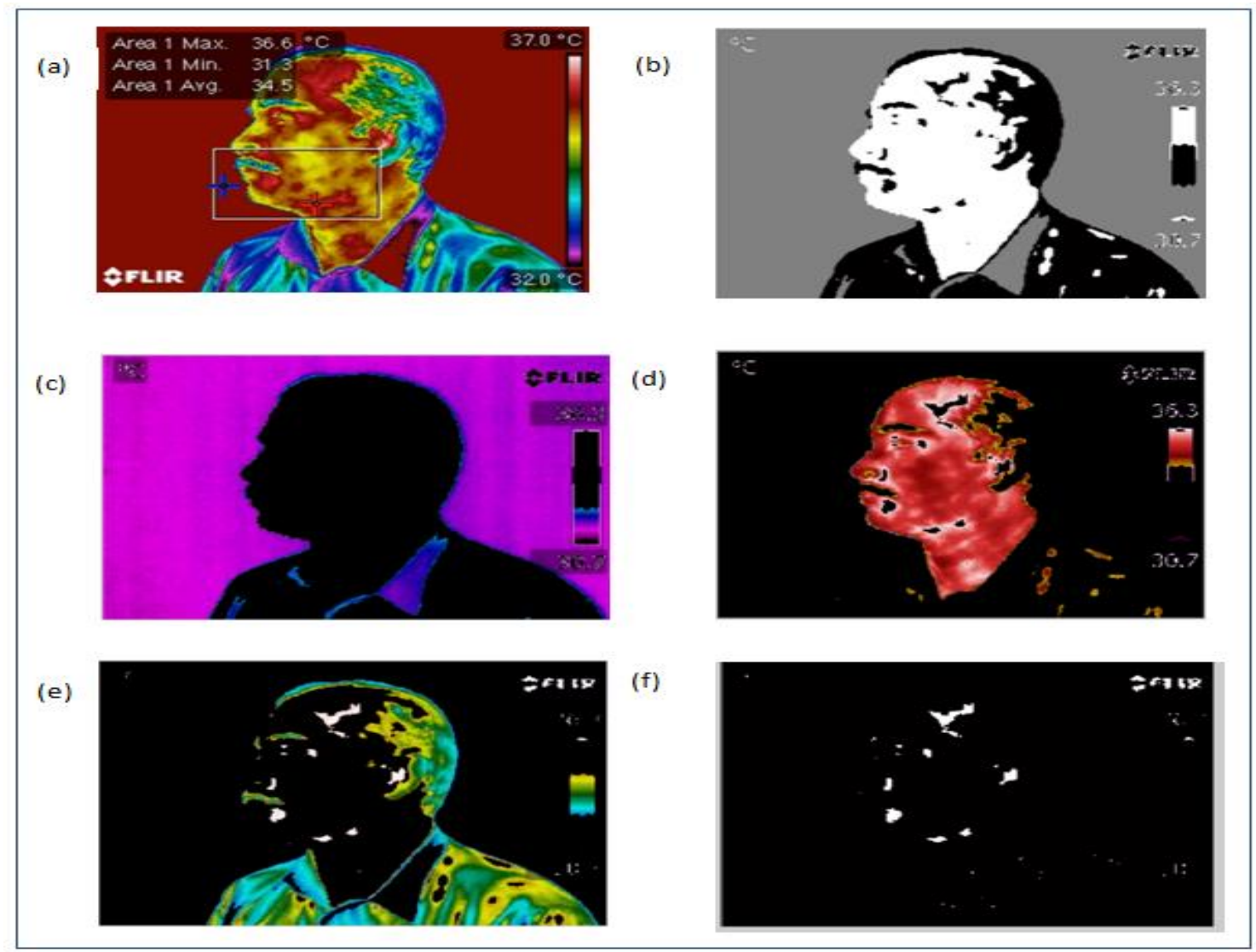

Fig. 5: Diseased Subject Front Profile (A) Input Thermal Image, (B) Image with Its Cluster Index, (C) Cluster 1 Depicting the Background, (D) Cluster 2 Depicting other Regions, (E) Cluster 3 Depicting High Temperature Regions, (F) Segmented Image Depicting Only High Temperature Regions.

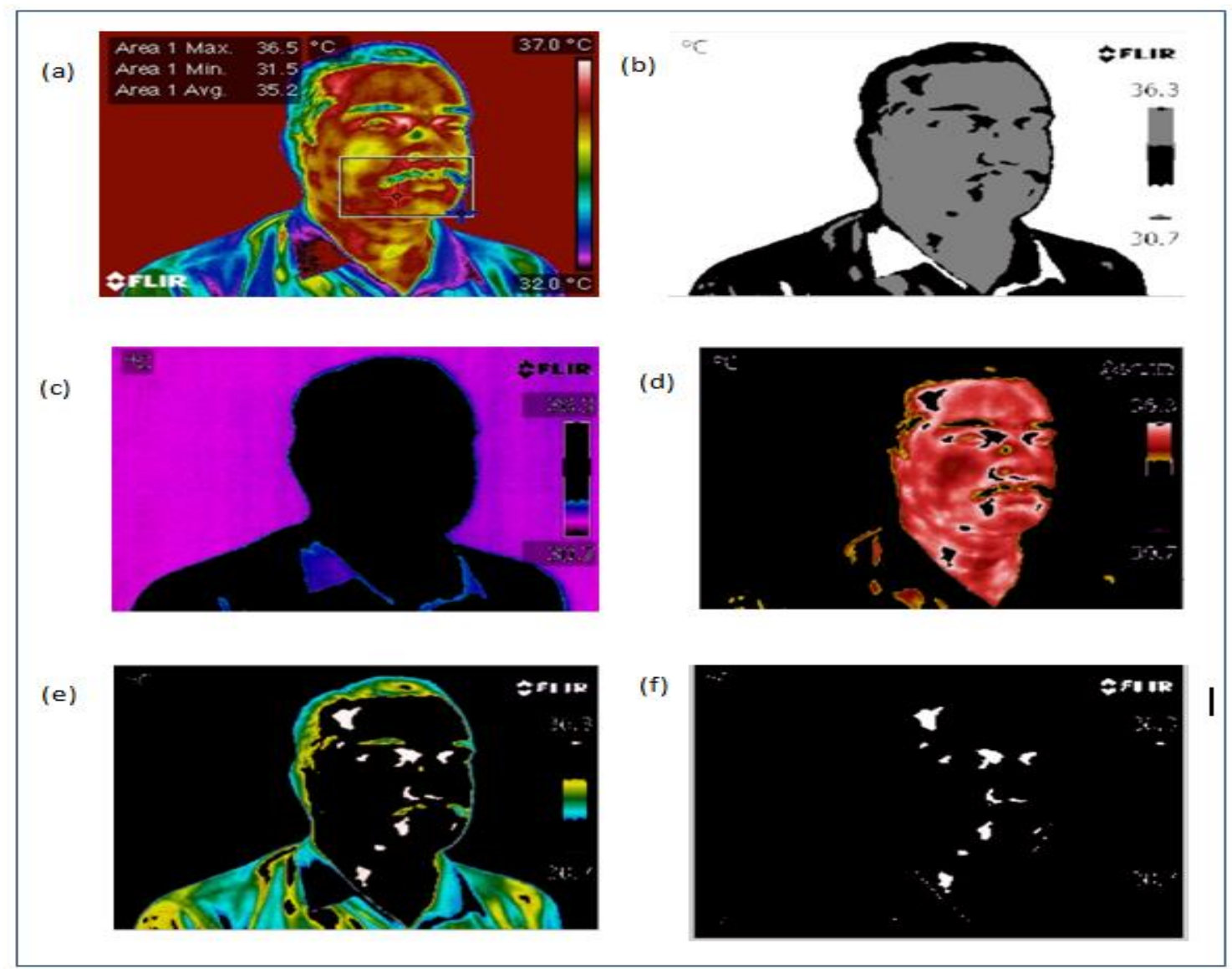

Fig. 6: Diseased Subject Right Profile (A) Input Thermal Image, (B) Image with Its Cluster Index, (C) Cluster 1 Depicting the Background, (D) Cluster 2 Depicting Other Regions, (E) Cluster 3 Depicting High Temperature Regions, (F) Segmented Image Depicting Only High Temperature Regions. 


\section{Discussion}

Early studies of functional disorders or dysfunction of the masticatory system focused on symptoms observed or anatomical evidences. The symptoms recorded as significant parameters were pain on movement of the mandible, muscle pain, swelling, etc. However, no such means of quantifying or even diagnosing asymptomatic diseases was researched upon. The current study has shown the value of infrared thermography as a technique which can be used for obtaining additional relevant information of patients with dental disorders, both symptomatic, and asymptomatic. In this study all abnormal subjects showed elevated temperatures over the affected region, which is mostly due to the increased muscular activity and therefore a reciprocal increase in blood supply.

Currently thermal imaging techniques were used for the evaluation of various dental disorders by qualitative and quantitative methods. This technique is also used as research tool in the field of dentistry for identifying the root canal inflammation, cavitations and in the development of abscess. Asymptomatic dental issues can be diagnosed using thermography, as seen in the study with patients showing no apparent problems or complaints about pain or discomfort. Compared to other imaging modalities, the thermal image interpretation is easier and faster method which is apparent from the images acquired during the study, similar to findings by Merla et al wherein they distinguished between healthy patients and patients suffering from myofascial pain using functional infrared imaging [13-15].

Gratt and his colleagues classified the patients with chronic orofacial pain as normal, hot or cold when the temperature in a selected anatomic area $(\Delta \mathrm{T})$ fell between certain assigned ranges [16], [17]. The current study reinforces the findings of the study made by Gratt et al, since temperature was found to be significantly elevated in subjects with dental issues in comparison with normal subjects. Dibai Filho et al. (2015) studied the reliability of different methodologies in infrared image analysis of myofascial trigger points in the upper trapezius muscles. They obtained excellent inter-rater reliability for point and line analysis with inter class correlation co-efficent equal to 0.908 and 0.918 respectively. They finally suggested that the infrared imaging technology was suitable for diagnosis of myofascial trigger points in the upper trapezius muscle in clinical and research practices [18]. Rodriguesbigaton et al. (2013) predicted the temporomandibular (TMD) disorder in arthralgia patients using thermal imaging technique with good accuracy. They found that the skin temperature was greater significance in the left side $(\mathrm{p}=0.004)$ compared to right side temporomandibular joint $(\mathrm{TMJ})(\mathrm{P}=.012)$ in arthralgia group They achieved excellent intra-rater and inter-rater reliability in the infrared image analysis of TMJ [19]. Haddad et al. (2014) conducted a preliminary study to test the efficacy of infrared thermal imaging in the characterization of masticatory muscle region in volunteers with and without the myogenic temporomandibular disorder. The temperature measured at masseter and anterior temporalis muscle regions in myogenic TMD volunteers $\left(32.85 \pm 0.85^{\circ} \mathrm{C}\right.$ and $34.37 \pm 0.64^{\circ} \mathrm{C}$, respectively) were significantly lower than the controls $\left(33.49 \pm 0.92^{\circ} \mathrm{C}\right.$ and $34.78 \pm 0.44^{\circ} \mathrm{C}$, respectively).They also obtained the sensitivity $(70 \%)$ and specificity (73\%) by using roc curves in the masseter region. They also suggested that the infrared thermal imaging was used as the complimentary tool in the diagnosis of TMD [20].

$\mathrm{X}$-rays indeed do identify infections to an extent, but it could not be able to predict the chronic heat inflammation and infection as easily. The advantages of thermal imaging were to locate areas of chronic heat before the challenged site gets severely infected. The temperature measurement, using thermal cameras, is affected by a wide range of factors, that is, room temperature, humidity, ventilation, and the presence of external heat sources. Therefore, in our study all stages of thermographic assessment were performed in the same environmental conditions [14]. Several studies related to infrared thermography for dental examination provided and additional insight into the patients oral and dental condition, aiding the process of examination, which is also implied by the study. From this data it is safe to infer that, presence of infections, significantly increases the temperature of the region, which, is in coherence with other previously conducted studies [21-24].

\section{Conclusion}

From this study, we can infer that with the incidence of dental abnormalities, the skin surface temperature of the body, that is, the region of interest, is elevated. This elevation is significantly apparent in the mean of minimum and maximum temperatures taken from the region of interest that is front, left and right of the face. The images also depict visual differences between normal and diseased subjects. In future, other advanced algorithms will be implemented and results will be compared with the results obtained using K-means algorithm. The acquired temperature values and feature extracted values will be given as an input to three different neural networks and the performance of the networks will be compared based on accuracy and sensitivity. In this study, facial thermograms were segmented using k-means algorithm and hence thermography could be used as complementary diagnostic tool for detection of asymptomatic and symptomatic dental diseases.

\section{References}

[1] Tonetti MS, Jepsen S, Jin L, Otomo-Corgel J. Impact of the global burden of periodontal diseases on health, nutrition and wellbeing of mankind: A call for global action. J Clin Periodontol. 2017; 00:1-7. https://doi.org/10.1111/jcpe.12732.

[2] Shewale AH, Gattani DR, Bhatia N, Mahajan R, Saravan SP. Prevalence of Periodontal disease in the general population in India-A systematic Review. J Clin Daign Res 2016; 10(6): ZE04-ZE09 https://doi.org/10.7860/JCDR/2016/17958.7962.

[3] http://www.who.int/mediacentre/factsheets/fs318/en/

[4] Abesi F, Mirshekar A, Moudi E, Seyedmajidi M, Haghanifar S, Haghighat N, Bijani A. Diagnostic Accuracy of digital and conventional radiography in the detection of non-cavitated approximal dental caries. Iran J Radiol 2012; 9(1): 17-21 https://doi.org/10.5812/iranjradiol.6747.

[5] Gomez J. Detection and diagnosis of the early caries lesion.BMC Oral Health 2015; 15 (Suppl 1): S3 https://doi.org/10.1186/14726831-15-S1-S3.

[6] Snekhalatha U, Sowmiya V, Nilkantha G. A computer aided diagnostic based Thermal image Analysis: A potential tool for the Evaluation of Rheumatoid Arthritis in Hand. Journal of Medical and Biological Engineering, on line: $30^{\text {th }}$ September 2017, DOI https://doi.org/10.1007/s40846-017-0338-x.

[7] Gratt BM, Anbar M. Thermology and facial telethermography: Part II: Current and future clinical applications in dentistry. Dento maxillofacial Radiology. 27: 68-74, 1998. https://doi.org/10.1038/sj.dmfr.4600324.

[8] Gratt BM, Graff-Radford SB, Shetty V, Solberg WK, Sickles EA A six-year clinical assessment of electronic facial thermography Dentomaxillofacial Radiology. 25: $247 \quad-255, \quad 1996$ https://doi.org/10.1259/dmfr.25.5.9161178

[9] Hayase Y, Wakasa T, Uemura M, Adachi K, Ochi S and Kishi K. Clinical Evaluation of Thermography in the Diagnosis of Malignan Tumors in the Oral and Maxillo-Facial Region. Oral Radiology 8(1):1-6, 1992. https://doi.org/10.1007/BF02347273.

[10] Durnovo EA, Potekhina YP, Marochkina MS, Yanova NA, Sahakyan MY, Ryzhevsky DV. Diagnostic capabilities of Infrared thermography in the examination of patients with diseases of maxillofacial area. Clinical Medicine 2014; 6(2): 61-65

[11] Pogrel MA, Yen CK, Taylor RC. Infrared thermography in oral and maxilla facial surgery. Oral surgery orla medicine oral pathology. 1989; 67(2): 126-31 https://doi.org/10.1016/0030-4220(89)90315$\underline{0}$.

[12] Snekhalatha U, Anburajan.M, Sowmiya V, Venkatraman.B, Menaga.M. Automated hand thermal image segmentation and feature ex- 
traction in evaluation of rheumatoid arthritis" Proceedings of the Institution of Mechanical Engineers, Part H: Journal of Engineering in $\quad$ Medicine, 2015; 229(4):319-331 https://doi.org/10.1177/0954411915580809.

[13] Merla A, Ciuffolo F, D'Attilio M, Tecco S, Festa F, De Michele G, Tangherlini A, Romani GL; Functional Infrared Imaging in the Diagnosis of the Myofascial Pain.

[14] Moinuddin Hassan, Tyuichi Kimura, Jcakimasa Asai, Akihiro Shimase,Masakazu Fukuoka and Ttatsuo Togawa; Imaging Of Skin Thermal Properties By Changing Ambient Radiation TemperatureAn Electrical Control System For Stepwise Change In Ambient Radiation temperature; Engineering in Medicine and Biology Society, $1-3 ; 1998$.

[15] Sarbani Deb Sikdar, Anshul Khandelwal, Savita Ghom, Rajkumar Diwan, FM Debta: Thermography: A New Diagnostic Tool in Dentistry. Journal of Indian Academy of Oral Medicine and Radiology 22(4):206-210, October-December 2010.

[16] Gratt BM, Sickles EA, Ross JB. Thermographic characterization of an internal derangement of the temporomandibular joint. Journal of Orofacial Pain 8: 197-206, 1994.

[17] Gratt BM, Sickles EA, Wexler CA. Thermographic characterization of osteoarthrosis of the temporomandibular joint. Journal of Orofacial Pain. 7: 345-353, 1993. https://doi.org/10.1590/bjptrbf.2014.0076.

[18] Dibai-Filho AV, Guirro EC, Ferreira VT, Brandino HE, Vaz MM, Guirro RR (2015). Reliability of different methodologies of infrared image analysis of myofascial trigger points in the upper trapezius muscle. Braz J Phys Ther 19: 122-128

[19] Rodrigues-Bigaton D, Dibai Filho AV, Costa AC, Packer AC, de Castro EM (2013). Accuracy and reliability of infrared thermography in the diagnosis of arthralgia in women with temporomandibular disorder. J Manipulative Physiol Ther. 36:253-8. https://doi.org/10.1016/j.jmpt.2013.04.006.

[20] Haddad DS, Brioschi ML, Vardasca R, Weber M, Crosato EM, Arista ES (2014). Thermographic chraracterization of masticatory muscle regions in volunteers with and without myogenous temporomandibular disorder: preliminary results. Dentomaxillofacial radiology 43: 20130440. https://doi.org/10.1259/dmfr.20130440.

[21] Komoriyama M, Nomoto R, Tanaka R, Hosoya N, Gomi K, Iino F, Yashima A, Takayama Y, Tsuruta M, Tokiwa H, Kawasaki K, Arai T, Hosoi T, Hirashita A, Hirano S. Application of Thermography in Dentistry-Visualization of Temperature Distribution on Oral Tissues. Dental Material Journal 22(4):1-7, 2003. https://doi.org/10.4012/dmj.22.436.

[22] Gratt BM, Sickles EA. Electronic facial thermography: an analysis of asymptomatic adult subjects. J Orofacial Pain. 9: 255- 265, 1995.

[23] McBeth SA, Gratt BM. A cross-sectional thermographic assessment of TMJ problems in orthodontic patients. American Journal of Orthodontics and Dentofacial Orthopedics. 109: 481-488, 1996. https://doi.org/10.1016/S0889-5406(96)70132-4.

[24] Biagioni PA, Longmore RB, McGimpsey JG, Lamey PJ. Infrared thermography. Its role in dental research with particular reference to craniomandibular disorders. Dentomaxillofacial Radiology. 25: 119-124, 1996. https://doi.org/10.1259/dmfr.25.3.9084259.

[25] S.V.Manikanthan and V.Rama“Optimal Performance of Key Predistribution Protocol In Wireless Sensor Networks" International Innovative Research Journal of Engineering and Technology, ISSN NO: 2456-1983, Vol-2, Issue-Special-March 2017.

[26] T. Padmapriya, V.Saminadan, "Performance Improvement in long term Evolution-advanced network using multiple imput multiple output technique", Journal of Advanced Research in Dynamical and Control Systems, Vol. 9, Sp-6, pp: 990-1010, 2017.

[27] N Bala Dastagiri, K Hari Kishore "Novel Design of Low Power Latch Comparator in 45nm for Cardiac Signal Monitoring", International Journal of Control Theory and Applications, ISSN No: 0974-5572, Vol No.9, Issue No.49, page: 117-123, May 2016. 